\title{
Controle glicêmico à distância dos idosos diabéticos insulinizados: uma experiência da atuação do Núcleo de Apoio à Saúde da Família (NASF) em tempos de pandemia da COVID-19
}

\author{
Hélida Maria de Lima Maranhão Brasileiro, Conciana Duarte Monte, Washington José dos \\ Santos, Mateus do Amaral Meira, Ana Nery Xavier Pereira, Rosa Luiza de Lima Ramos, \\ Rafaela Nascimento, Ana Fabrícia Morais, Emília Maria Carvalho Feitosa
}

\section{RESUMO}

O Diabetes Mellitus (DM) é um importante problema de saúde mundial. Com o aumento da expectativa de vida, aumenta-se a prevalência da doença com suas complicações crônicas nos idosos. 0 tratamento efetivo do DM com insulina é complexo e difícil de ser realizado corretamente pelo idoso, pois requer a execução de várias etapas para o preparo e aplicação do medicamento. $O$ Núcleo de Apoio à Saúde da Família (NASF), dentro da sua dimensão clínico-assistencial, realiza educação de saúde em DM, visando práticas seguras para o manejo correto da insulina por parte dos idosos/cuidadores. Com a pandemia da COVID-19, o telemonitoramento foi a estratégia de trabalho utilizada pelo NASF para promover o controle glicêmico dos idosos à distância. A avaliação dos teleatendimentos identificou erros no preparo e aplicação da insulina, verificou o perfil glicêmico dos idosos, permitiu estratificação de risco desses pacientes e reforçou medidas necessárias para a corresponsabilização do cuidado.

Palavras-chave: Núcleo de Apoio à Saúde da Família, Diabetes Mellitus, idosos, telemonitoramento, COVID-19.

\section{ABSTRACT}

Diabetes Mellitus (DM) is an important global health problem. Increased life expectancy leads to a higher prevalence of elderly diabetics with associated chronic complications. The effective treatment of DM with insulin is complex and difficult to be correctly performed by the elderly, as it requires the execution of several steps for insulin preparation and application. The Family Health Support Center (NASF), within its clinical-assistance dimension, conducts health education in DM, aiming at safe practices for the insulin correct handling by the elderly or their caregivers. The COVID-19 pandemic brought telemonitoring was NASF's work strategy to promote elderly glycemic control at a distance. The service evaluation identified errors in the insulin preparation and application, verified the elderly glycemic profile, allowed risk stratification of these patients and reinforced necessary measures for the co-responsibility of elderly care..

Keywords: Family Health Support Center, Diabetes Mellitus, elderly, telemonitoring, COVID-19.

Revista da Rede APS 2021

Publicada em: 28/12/2021

DOI:10.14295/aps.v3i3.201

Hélida M. L. M. Brasileiro (Universidade Federal Rural de Pernambuco, Recife, PE, Brasil)

Conciana Duarte Monte (Prefeitura da cidade do Recife, Recife, PE, Brasil)

Washington José dos Santos (Prefeitura da cidade do Recife, Recife, PE, Brasil)

Mateus do Amaral Meira (Prefeitura da cidade do Recife, Recife, PE, Brasil)

Ana Nery Xavier Pereira (Prefeitura da cidade do Recife, Recife, PE, Brasil)

Rosa Luiza de Lima Ramos (Prefeitura da cidade do Recife, Recife, PE, Brasil)

Rafaela Nascimento (Prefeitura da cidade do Recife, Recife, PE, Brasil)

Ana Fabrícia Morais (Prefeitura da cidade do Recife, Recife, PE, Brasil)

Emília Maria Carvalho Feitosa (Prefeitura da cidade do Recife, Recife, PE, Brasil)

Correspondência para: Hélida M. L. M. Brasileiro helida.maranhao@ufrpe.br 


\section{INTRODUÇÃO}

O diabetes mellitus (DM) consiste em um distúrbio metabólico caracterizado por hiperglicemia persistente decorrente da deficiência na produção de insulina ou na sua ação, ou em ambos os mecanismos. A classificação do DM baseia-se na sua etiologia, assim, tem-se o DM tipo 1 como uma doença autoimune, poligênica, decorrente da destruição das células beta-pancreáticas; o DM tipo 2 que está relacionado à perda progressiva da secreção insulínica combinada com resistência à insulina; o diabetes gestacional caracterizado pela hiperglicemia de graus variados diagnosticada durante a gestação, na ausência de critérios de DM prévio e, por fim, outros tipos DM secundários à fatores monogênicos, endocrinopatias, infecções e uso de medicamentos (AMERICAN DIABETES ASSOCIATION, 2019). Desse modo, a insulina exógena é sempre utilizada no tratamento do DM tipo 1, mas também pode ser utilizada no diabetes gestacional e no DM tipo 2 quando o pâncreas passa a produzir quantidades insuficientes do hormônio.

Em 2017, o Brasil foi o 4o país no mundo com o maior número de pessoas entre 20 e 79 anos com diabetes, totalizando 12,5 milhões de indivíduos (INTERNATIONAL DIABETES FEDERATION, 2017). A população idosa se destaca, dentro desse contexto, por apresentar particularidades que devem ser consideradas na escolha do melhor esquema terapêutico para evitar ou retardar o surgimento de complicações.

O DM está associado a maiores taxas de hospitalizações, maior utilização dos serviços de saúde, bem como maior incidência de doenças cardiovasculares e cerebrovasculares, cegueira, insuficiência renal e amputações não traumáticas de membros inferiores. Existem evidências de que indivíduos com diabetes mal controlado ou não tratado desenvolvem mais complicações do que àqueles com o diabetes bem controlado, por isso, é importante que o diabético tenha um adequado controle glicêmico (SOCIEDADE BRASILEIRA DE DIABETES - SBD, 2019).
Buscando promover a qualidade de vida desses pacientes, aliviar a sobrecarga nos sistemas de saúde e evitar possíveis hospitalizações de pacientes com diabetes descompensado, surgiu a educação de saúde em diabetes (SBD, 2019). 0 Núcleo de Apoio à Saúde da Família (NASF), dentro de sua dimensão clínico-assistencial, atua individualmente, de forma compartilhada e em grupos educativos com a autogestão do DM, visando principalmente o manejo correto e seguro da insulina por parte dos pacientes idosos e/ou cuidadores. Isso torna-se relevante porque a educação em diabetes é considerada parte do próprio tratamento da doença e, por isso, deve ser inserida em todos os níveis de assistência ao usuário (FRID et al., 2016).

Com a pandemia da COVID-19, a população que sofre de comorbidades crônicas precisa de especial atenção nesse momento, uma vez que devem continuar seus tratamentos e são os mais atingidos fatalmente pela doença. O risco de agravamento da COVID-19 está aumentado em pacientes diabéticos, por isso o controle glicêmico adequado pode atenuar o risco de complicações (SILVA; ARAÚJO, 2020). As etapas para o preparo e aplicação da insulina, imprescindíveis no tratamento do DM tipo 1 , são cheias de particularidades e requerem um acompanhamento regular desses pacientes a fim de garantir a adesão e efetividade do tratamento prescrito.

O controle glicêmico do idoso à distância, através do telemonitoramento, foi a alternativa adotada pela equipe NASF para continuar o acompanhamento desses pacientes e, promover assim, a corresponsabilização do cuidado.

\section{MÉTODO}

\section{Tipo e Local do Estudo}

O estudo conduzido foi do tipo relato de experiência do NASF no monitoramento à distância dos níveis glicêmicos de idosos diabéticos com insulinoterapia prescrita e assistidos pela equipe 2 da Estratégia de Saúde da Família (ESF) Bernard Van Leer, localizada no 
bairro de Brasília Teimosa - DS VI, na cidade do Recife-PE.

\section{Amostra}

Os idosos diabéticos com tratamento insulínico prescrito, cadastrados no grupo de Hipertensos e Diabéticos (Hiperdia) da equipe de saúde e que participavam voluntariamente do grupo de educação em saúde denominado Insulinizados, conduzido pelo NASF na ESF, tiveram seus níveis glicêmicos monitorados à distância entre os meses de abril e maio de 2020 através do teleatendimento.

\section{Grupo de educação em saúde}

O grupo denominado Insulinizados contava com a participação de 48 idosos diabéticos que faziam uso de insulina e/ou seus cuidadores. Os encontros ocorriam regularmente na unidade no período pré-pandemia e consistiam, principalmente, em orientações práticas quanto aos aspectos seguros para preparo e aplicação das insulinas NPH e regular tanto na apresentação de frascos-ampola quanto na de caneta, seguindo os protocolos recomendados pela Sociedade Brasileira de Diabetes.

O grupo focava principalmente na promoção da efetividade terapêutica do tratamento insulínico, mas também trazia temas relacionados aos cuidados com o pé diabético, aspectos nutricionais, emocionais e sociais envolvidos com a doença, uma vez que a equipe era composta por farmacêutica, fonoaudióloga, fisioterapeuta, terapeuta ocupacional, nutricionista, psicólogo e assistente social.

\section{Protocolo de monitoramento} glicêmico à distância

Foram elaboradas planilhas de acompanhamento para cada idoso e divididas em 04 blocos de atenção que eram checados durante o teleatendimento: a) etapas para o preparo e aplicação da insulina NPH e regular em frasco-ampola; b) etapas para o preparo e aplicação da insulina NPH e regular em caneta; c) outros aspectos relacionados à utilização da insulina como transporte, armazenamento, controle de validade, temperatura do medicamento durante a aplicação, assertividade da dose, rodízio das aplicações, a não reutilização das agulhas, descarte correto dos insumos, além do reforço das orientações quanto à prevenção de hipoglicemias; d) diário para registro glicêmico: os idosos foram incentivados a realizar o automonitoramento glicêmico pelo menos duas vezes ao dia (em jejum e ao deitar-se à noite) e registrar os níveis glicêmicos durante 15 dias para posterior coleta e avaliação dos dados. Os níveis glicêmicos foram analisados de acordo com Leroith et al. (2019), como mostrado na tabela abaixo:

Tabela 1 - Classificação dos níveis glicêmicos de idosos no jejum e ao deitar-se.

\begin{tabular}{|l|l|l|}
\hline \multicolumn{1}{|c|}{ Níveis glicêmicos } & \multicolumn{1}{|c|}{ Jejum (mg/dL) } & \multicolumn{1}{c|}{ Ao deitar-se $(\mathrm{mg} / \mathrm{dL})$} \\
\hline Hipoglicemia & $<70$ & $<70$ \\
\hline Normoglicemia & Entre 90 e 150 & Entre 100-180 \\
\hline Hiperglicemia & A partir de 151 & A partir de 181 \\
\hline
\end{tabular}

Fonte: LEROITH et al. (2019).

\section{Teleatendimentos realizados pela equipe NASF}

Para cada idoso foram realizados 02 teleatendimentos durante esse período, ocorrendo o segundo 15 dias após o primeiro. Nas ligações telefônicas, chamadas de voz ou vídeo realizadas (via aplicativo de WhatsApp), os idosos e/ou cuidadores foram questionados e suas respostas registradas quanto à execução 
das etapas para o preparo e aplicação da insulina NPH e regular tanto em frascos-ampola quanto em canetas, assim como quanto aos outros aspectos relacionados à utilização da insulina e à orientação quanto ao automonitoramento glicêmico por 15 dias. No segundo teleatendimento, além do reforço das informações anteriormente passadas, os registros glicêmicos foram coletados.

\section{RESULTADOS E DISCUSSÃO}

Dentre as etapas para preparo e manuseio das insulinas NPH e regular nas apresentações frascos-ampola e caneta, a homogeneização correta é imprescindível para a efetividade do medicamento e apenas metade dos idosos a realizavam corretamente. Além disso, $45 \%$ dos idosos que faziam uso de frascos-ampola de insulina não realizavam o procedimento que evita a formação de bolhas de ar na seringa antes de aspirar a insulina e para os que usavam caneta, $70 \%$ deles não comprovavam o fluxo da agulha antes da administração do medicamento, o que também compromete a efetividade do tratamento antidiabético.

Nos teleatendimentos de retorno, observou-se uma maior adesão à homogeneização correta da insulina (realizada por $82 \%$ dos idosos), ao cumprimento das etapas para evitar à formação de bolhas de ar na seringa (realizado por aproximadamente $55 \%$ deles) e à comprovação do fluxo de insulina (realizada por $50 \%$ dos idosos que usavam a caneta).

A avaliação das respostas sobre os outros aspectos relacionados à insulina, mostrou que 90\% dos idosos transportavam a insulina corretamente. Todos os idosos armazenavam adequadamente as canetas e os frascos-ampola de insulina lacrados na geladeira (prateleira acima da caixa de vegetais) assim como os frascos-ampola em uso. No entanto, todas as canetas de insulina que estavam em uso pelos idosos, continuavam a ser armazenadas na geladeira, sendo o recomendado à temperatura ambiente. De acordo com o fabricante, tal procedimento pode comprometer o registro da dose correta da insulina na caneta.
Nenhum dos idosos que utilizava frascosampola ou caneta controlava a validade da insulina após aberta, que é em torno de 4 semanas para frascos-ampola a partir do 10 dia de uso e 6 semanas para as canetas. Há diferenças nas formas de conservação e no tempo de validade entre a insulina em uso e a lacrada para que a potência e a estabilidade delas sejam mantidas. Por isso, deve-se anotar a data inicial de uso da insulina a fim de acompanhar sua validade, bem como verificar seu aspecto antes da utilização (SBD, 2019). Para os idosos que faziam uso de frascos-ampola, apenas $45 \%$ deles esperavam os $15-30$ minutos necessários para a insulina atingir a temperatura ambiente antes da aplicação e, assim, evitar dores e irritação durante o procedimento. Tal ação não se faz necessária para as canetas, uma vez que as em uso já são armazenadas na temperatura ambiente $\left(15^{\circ}-30^{\circ} \mathrm{C}\right)$.

A assertividade da dose, continuou a ser um problema constante como já identificado em atendimentos anteriores. Todos os idosos que usam principalmente os frascos-ampola de insulina e necessitam da seringa para aplicação tiveram dificuldades de enxergar nela não só os números, mas a escala que é graduada de $2 \mathrm{em}$ 2 unidades e só permite doses pares. Esse problema foi minimizado quando o idoso tinha um cuidador ou quando ele já estava em uso da insulina em caneta.

Dentre os locais recomendados para a aplicação de insulina, a região abdominal foi a preferida pela maioria dos idosos teleacompanhados. No entanto, nenhum deles realizava os rodízios de aplicação corretamente, o que levou ao relato de endurecimento e dor no local da aplicação, o que caracteriza a lipodistrofia. E como consequência, a absorção de insulina a partir desses locais torna-se imprevisível, podendo ocorrer hiperglicemia, hipoglicemias inexplicáveis e maior variabilidade glicêmica. Por isso, o rodízio das aplicações é decisivo para evitar o descontrole glicêmico (PIMAZONNINETO, 2017).

Os idosos reutilizavam a mesma agulha por até 2 aplicações de insulina NPH/dia e por até 3 aplicações de insulina regular diariamente. Essa 
prática está amparada na orientação do Caderno da Atenção Básica do Ministério da Saúde $n^{\circ}$. 36, que permite a reutilização das agulhas por até 8 vezes (BRASIL, 2013), mesmo não havendo essa recomendação pelo fabricante e nem pela Agência Nacional de Vigilância Sanitária, que classifica esse item como de uso único, pois pode causar prejuízos que comprometem a efetividade da terapia insulínica (PIMAZONNI-NETO, 2017). O descarte dos frascos-ampola, seringas, canetas, lancetas e fitas de glicosímetro era realizado corretamente por todos os idosos: em recipientes de plástico duro, com abertura larga, vedados com tampa. Eram entregues ao seu agente comunitário de saúde ou levados diretamente à unidade de Saúde da Família.

Figura 1 -Distribuição e classificação dos níveis glicêmicos dos idosos no jejum. Normoglicemia (entre 90-150 mg/dL), hiperglicemia (> $151 \mathrm{mg} / \mathrm{dL}$ ) e hipoglicemia $(<70 \mathrm{mg} / \mathrm{dL})$.

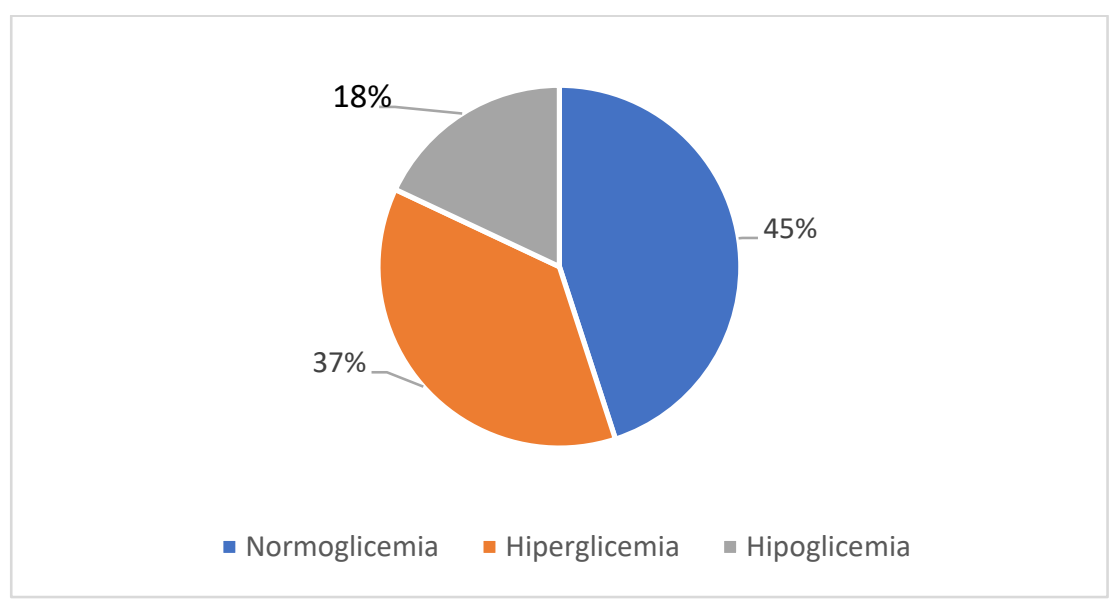

Fonte: LEROITH et al. (2019).

Figura 2 - Distribuição e classificação dos níveis glicêmicos dos idosos ao deitar-se. Normoglicemia (entre $100-180 \mathrm{mg} / \mathrm{dL}$ ), hiperglicemia (> $181 \mathrm{mg} / \mathrm{dL}$ ) e hipoglicemia $(<70 \mathrm{mg} / \mathrm{dL}$ ).

Fonte: LEROITH et al. (2019).

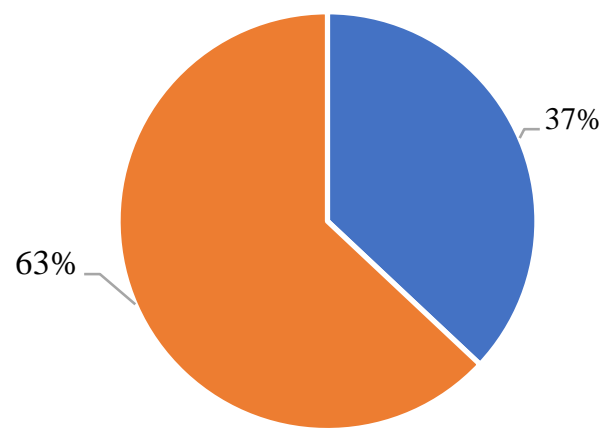

- Normoglicemia $\quad$ Hiperglicemia 
No teleatendimento de retorno, observou-se que a prática do rodízio entre as aplicações passou a ser executada, agora, por aproximadamente $40 \%$ dos idosos. No entanto, a principal dificuldade manteve-se em não conseguir a assertividade da dose, principalmente entre os usuários de frascosampola. A média dos registros glicêmicos em jejum e ao deitar-se durante o período de 15 dias são apresentados nas figuras 1 e 2, respectivamente.

Os dados mostraram que no jejum, $45 \%$ dos idosos estavam normoglicêmicos, 37\% hiperglicêmicos e $18 \%$ hipoglicêmicos. A hiperglicemia no jejum poderia estar associada ao "fenômeno do alvorecer", que consiste no aumento da glicemia nas primeiras horas da manhã (entre 05 e 08h), devido à liberação de hormônios contrarreguladores (adrenalina, glucagon, cortisol e hormônio do crescimento) durante a madrugada que estimulam a liberação de glicose pelo fígado e suprimem a ação da insulina, elevando a glicemia nessas primeiras horas (SBD, 2019).

Já a hipoglicemia poderia estar associada à administração de uma dose maior de insulina que a prescrita e/ou à diminuição na ingestão de alimentos. A hipoglicemia no idoso está relacionada a uma resposta autonômica deficiente associada ao déficit na secreção de hormônios contrarreguladores. No idoso, a hipoglicemia pode aumentar o risco de quedas além de poder vir acompanhada de lesões em órgãos vitais como cérebro e coração (SBD, 2019; NERY, 2008).

Ao deitar-se, a maioria dos idosos (63\%) estavam normoglicêmicos e o restante em hiperglicemia. Nenhum idoso apresentou hipoglicemia nesse período. A hiperglicemia também pode estar associada a aplicação de dose incorreta de insulina, excesso de alimentação, carência de exercícios físicos e até mesmo ao estresse causado por alguma comorbidade ou situação psicoemocional (SBD, 2019).

\section{CONSIDERAÇÕES FINAIS}

A insulina é um medicamento avaliado e classificado como potencialmente perigoso segundo o Instituto para Práticas Seguras no Uso de Medicamentos - ISMP (2017), pois apresenta risco aumentado de danos significativos em decorrência de falhas na sua utilização. A técnica correta para utilização da insulina é complexa, pois engloba desde o transporte, armazenamento, preparo e aplicação da insulina, rodízio adequado até o descarte correto dos resíduos. Por isso, é fundamental que uma equipe multiprofissional esteja capacitada na educação em diabetes para dar assistência adequada ao idoso e/ou cuidador a fim de promover a autonomia e a corresponsabilização do cuidado.

As canetas para aplicação de insulina têm se tornado uma opção popular nos últimos anos. Em 2019, o Sistema Único de Saúde iniciou a dispensação de caneta de insulina NPH e regular descartáveis para pacientes de até 15 anos e para idosos a partir dos 60 anos (BRASIL, 2019), uma conquista para o tratamento insulínico no Brasil. A praticidade do manuseio, transporte, disponibilidade de agulhas mais curtas assim como as de alto fluxo, o registro de doses pares e ímpares, podendo-se ainda registrar doses de $1 / 2$ em $1 / 2$ unidade são algumas das vantagens da caneta em relação à seringa. Isso permite maior aceitação social, adesão ao tratamento, assertividade das doses e, assim, maior controle glicêmico (SBD, 2019). A substituição dos frascos-ampolas por canetas de insulinas NPH e regular já é uma realidade para os idosos da atenção primária da cidade do Recife e ocorrerá gradualmente à medida que novas prescrições médicas forem dispensadas.

O telemonitoramento dos idosos diabéticos insulinizados teve uma boa receptividade por parte deles, inclusive por fazerem parte de um grupo com comorbidade crônica cuja uma possível infecção pelo coronavírus poderia ser agravada, principalmente se os níveis glicêmicos estivessem descompensados. O controle glicêmico diário não é uma tarefa fácil, pois muitos idosos têm dificuldades de enxergar, moram sozinhos, são analfabetos e, muitas 
vezes, não aderem ao tratamento seja por esquecimento ou por não saber utilizar corretamente a insulina.

Desse modo, a incorporação do telemonitoramento à prática clínica da equipe NASF tornou-se uma ferramenta importante para o acompanhamento e controle glicêmico do idoso à distância. Essa estratégia de cuidado sinalizou sua viabilidade para períodos póspandemia da COVID-19, pois teve um impacto positivo na cogestão da doença: permitiu o aperfeiçoamento do controle glicêmico tanto pela equipe de saúde quanto pelo usuário, estratificou o risco desses pacientes possibilitando o planejamento do cuidado continuado, além de ratificar as práticas seguras para o preparo e aplicação da insulina, etapas imprescindíveis para que o controle glicêmico torne-se uma realidade no tratamento insulínico de idosos.

No entanto, esse acompanhamento só se tornou possível porque uma equipe de saúde multidisciplinar estava comprometida e engajada, adotando como uma das estratégias de cuidado a educação em diabetes. Educar o diabético e/ou seu cuidador é um trabalho continuado, pois requer capacitação dos profissionais de saúde, acompanhamento longitudinal com atendimentos regulares para que eles consigam realizar o autogerenciamento da doença na sua rotina.

A educação em diabetes abrange não só os aspectos relacionados à insulina como transporte, armazenamento, aplicação e descarte corretos, mas também foca na apropriação da doença pelos pacientes que passam a reconhecer e evitar sinais e sintomas que caracterizam quadros de hipo ou hiperglicemia, situações de descompensação glicêmica que aceleram as complicações da doença ao longo prazo. Além disso, um impacto positivo sobre as comorbidades (hipertensão arterial, obesidade e dislipidemia) também pôde ser observado, mesmo que a avaliação desses parâmetros não tenham sido objetivo do presente trabalho.

Apesar de ser um desafio diário para a equipe de saúde monitorar a glicemia dos idosos diabéticos durante o tratamento insulínico, observou-se que a participação de uma equipe multiprofissional capacitada e atividades de educação em saúde aliadas ao telemonitoramento foram capazes de promover a continuidade do cuidado, principalmente durante a pandemia da COVID-19, quando os atendimentos presenciais nas unidades de saúde estavam voltados para os casos emergenciais. Monitorar o idoso diabético insulinizado poderá diminuir o surgimento de complicações, o número de hospitalizações e viabilizar a corresponsabilização da gestão da doença, tornando a equipe mais próxima e presente no tratamento desses comunitários. 


\section{REFERÊNCIAS}

AMERICAN DIABETES ASSOCIATION. Classification and Diagnosis of Diabetes: Standards of Medical Care in Diabetes-2019. Diabetes Care. 2019; 42 (1): 13-28.

BRASIL. Ministério da Saúde. CGAFB/DAF/SCTIE/MS. Nota técnica no 204/2019. Informa sobre distribuição e critérios para dispensação das canetas aplicadores de insulinas NPH e Regular e agulhas para caneta aplicadora. [internet]. Disponível em: http://saude.sc.gov.br/index.php/informacoes-geraisdocumentos/vigilancia-em-saude/assistencia-farmaceutica/ 15525-nota-tecnica-n-204-2019-cgafb-dafsctie-ms/file. Acesso em 25 junho de 2020.

BRASIL. Ministério da Saúde. Secretaria de Atenção à Saúde. Departamento de Atenção Básica. Estratégias para o cuidado da pessoa com doença crônica: diabetes mellitus/Ministério da Saúde, Secretaria de Atenção à Saúde. Cadernos de Atenção Básica, $n^{\circ}$. 36. Departamento de Atenção Básica. Brasília: Ministério da Saúde, 2013. 160p.

FRID A.H.; KREUGEL G.; GRASSI G.; HALIMI S.; HICKS D.; HIRSCH L.J. New insulin delivery recommendations. Mayo Clinic Proceedings. 2016; 91 (9): 1231-1255.

INSTITUTE FOR SAFE MEDICATION PRACTICES (ISMP). ISMP Guidelines for Optimizing Safe Subcutaneous Insulin use in adults [internet]; 2017. Available from: https://www.ismp.org/sites/default/files/ attachments/2017-11/ISMP138-Insulin\%20Guideline- -051517-2-WEB.pdf. Acesso em 25 de junho 2020.

INTERNATIONAL DIABETES FEDERATION. IDF Atlas. 8. ed. Bruxelas: International Diabetes Federation; 2017.

LEROITH D.; BIESSELS G.J.; BRAITHWAITE S.S. et. al. Treatment of Diabetes in Older Adults: An Endocrine Society* Clinical Practice Guideline. The Journal of Clinical Endocrinology and Metabolism. 2019; 104 (5): 1520-1574.

NERY M. Hipoglicemia como fator complicador no tratamento do Diabetes Melito tipo 1. Arquivos Brasileiros de Endocrinologia \& Metabologia. 2008; 52 (2): 288-298.

PIMAZONI-NETTO A. (coordenador). Posicionamento oficial SBD no 01/2017: recomendações sobre o tratamento injetável do diabetes: insulinas e incretinas [Internet]. São Paulo: Sociedade Brasileira de Diabetes; 2017. Acesso em 24 de junho de 2019.

SILVA L.M.C.; ARAÚJO J.L. Clinical and community pharmacist's role in the COVID-19 pandemic. Research, Society and Development. 2020; 9 (7): 1-14.

SOCIEDADE BRASILEIRA DE DIABETES (SBD). Diretrizes da Sociedade Brasileira de Diabetes 2019-2020. São Paulo: Clannad Editora Científica, 2019. 